patient to be treated using this approach. An 84-year-old woman with intractable hydrocephalus following subarachnoid hemorrhage underwent an external ventricular drain clamping which was not tolerated owing to intracranial pressure (ICP) reaching $44 \mathrm{cmH}_{2} \mathrm{O}$. On the following day, her drain was clamped eight hours prior to undergoing successful endovascular deployment of the eShunt device with post-implant CT head showing no hemorrhage. Within 90 mins of eShunt insertion, the patient's ICP decreased from 38 to $<20$ $\mathrm{cmH}_{2} \mathrm{O}$ to followed by subsequent resolution of ventriculomegaly. This is the first patient treated for communicating hydrocephalus using a novel endovascular CSF shunt without the need for a burr hole, brain penetration or multiple skin incisions. This novel and unique percutaneous transluminal access to the central nervous system ushers a new pathway for noninvasive treatment of hydrocephalus and potential for percutaneous access to the central nervous system for intervention against neurological disorders.

Disclosures P. Lylyk: 1; C; Cerevasc. I. Lylyk: None. C. Bleise: None. J. Lundquist: None. E. Scrivano: None. B. Beneduce: 5; C; Employee Cerevasc. C. Heilman: 4; C; Stockholder. A. Malek: 4; C; Stockholder.

\section{LB-002 OPHTHALMIC ARTERY ANGIOPLASTY FOR AGE RELATED MACULAR DEGENERATION}

${ }^{1}$ I Lylyk ${ }^{*},{ }^{1} \mathrm{C}$ Bleise, ${ }^{2} \mathrm{~A}$ Francone, ${ }^{2} \mathrm{M}$ Charles, ${ }^{2} \mathrm{~T}$ Zompa, ${ }^{1} \mathrm{P}$ Lylyk. ${ }^{1}$ Insituto Medico ENER/Clinica La Sagrada Familia, Bs As, Argentina; ${ }^{2}$ Centro Oftalmológico Dr. Daniel Charles S.A, Buenos Aires, Argentina, Bs As, Argentina

10.1136/neurintsurg-2021-SNIS.243

Background Age-related macular degeneration (AMD) is the leading cause of irreversible blindness among the elderly in the developed world, for which there is currently no available treatment. Compromised ocular microcirculation due to aging and vascular disease contribute to retinal dysfunction and vision loss; decreased choroidal perfusion is evident in eyes with dry AMD. In an effort to evaluate whether or not the the ophthalmic artery (OA) is a contributor to decreased retinal perfusion in eyes with AMD, a study was conducted utilizing a 7T MRI and a custom built 32 channel head receive array coil with a birdcage transmit coil to non-invasively capture hemodynamic data of both arteries The study demonstrated a statistically significant decrease in both lumen diameter $(p=0.006)$ and volumetric flow rates $(p=0.041)$, and an increase in resistive index $(\mathrm{p}<0.001)$, which measures dynamic flow properties, in the OA of AMD patients as compared to age matched controls without AMD. These findings indicate the changes in or around the OA, may be contributing to decreased retinal perfusion in AMD patients, and present a potential treatment target in a patient population without therapeutic options.

Methods Five patients who presented late-stage AMD as evidenced by the presence of geographic atrophy or stabilized history of choroidal neovascularization, best corrected visual acuity (BCVA) of 20/400 or worse, in combination with processed intraarterial cone beam-volume CT images regarding the precise $\mathrm{OA}$ origin and the relationship with an atheroma plaque nearby or in the artery were selected. The eye with the worst visual acuity was chosen for treatment.

Results After a difficult cannulation of the OA due to its narrowing, balloon angioplasty was successfully performed in all of the five cases. Subjective patient reports indicated all patients perceived a benefit following the procedure; however, improved postoperative visual acuity did not confirm that perceived benefit for one of the patients. Mean preoperative BCVA was 20/710 and improved to 20/383 at 1 week postoperative, representing a mean gain of nearly 3 lines, remaining stable through month six.

Conclusion Our preliminary experience in a small cohort of patients suggest that OA angioplasty may be performed safely and there is subjective evidence of efficacy. The OA is a viable target to increase blood flow to the eye, restore retinal perfusion, and perhaps disrupt the disease process of AMD. To proceed with a randomized controlled trial, devices specific to this application should be developed.

Disclosures I. Lylyk: None. C. Bleise: None. A. Francone: None. M. Charles: None. T. Zompa: None. P. Lylyk: 2; C; OcuDyne.

\section{LB-003 TRANSRADIAL VERSUS TRANSFEMORAL ARTERIAL APPROACH FOR CEREBRAL ANGIOGRAPHY AND THE FREQUENCY OF SILENT EMBOLI ON DIFFUSION- WEIGHTED MRI}

V Carraro do Nascimento*, H Rice, L De Villiers. Gold Coast University Hospital, Southport, Australia

\subsection{6/neurintsurg-2021-SNIS.244}

Introduction The radial artery approach has become popular as 'radial-first' strategy for arterial access in neuroangiography and neurointerventions. ${ }^{1}$ Recent studies have shown that transradial arterial access (TRA) for cerebral angiography has been associated with reduced access site complication rates and improved patient satisfaction compared with transfemoral access (TFA). ${ }^{2} 3$ In spite of the increased adoption of the TRA technique, as well as proven decreased complication rates in the cardiology literature compared to TFA, the frequency of MRI diffusion-weighted imaging changes post cerebral angiography has not been described. It is known that diagnostic angiography, either TRA or TFA, of the cerebral vessels harbours a small but definite risk of neurological complications, such as a new focal neurological deficit, ${ }^{4}{ }^{5}$ ranging from $0 \cdot 55 \%$ to $3 \cdot 2 \% .^{6}$ The goal of this study was to evaluate the presence of abnormal diffusion restriction foci, which represents silent embolic events (SEE) during digital subtraction angiography (DSA) and correlated their presence and frequency with TRA or TFA.

Methods We analysed 120 consecutive adult diagnostic cerebral angiograms performed over 6 months, from January 2021 and concluding in June 2021, at a single tertiary centre, performed by a single operator. Access site was randomised aiming for approximately 50\% TRA and 50\% TFA by the end of the allocation period.

Results Of the 120 consecutive diagnostic cerebral angiograms, 58 (49\%) were performed via TRA, and 62 (51\%) were performed via TFA. One case was performed via right ulnar artery access (which we considered as TRA for the purpose of statistical analysis). Of the TRA cerebral angiograms, 8 demonstrated DWI changes (silent embolic events). Of the TFA procedures, one showed abnormal diffusion restriction. Two cases were converted from TRA to TFA, one due to a radial loop and one due to an incidental right aberrant subclavian artery. No vascular access site complications were observed. The mean number of vessels catheterised in the TRA group was 
2.3 and in the TFA group was 2.5. The mean procedure time in the TRA group was 20 and in the TFA group was 18 minutes. No patient developed neurological symptoms.

Statistical analysis were concluded using StataCorp. 2017. Stata Statistical Software: Release 15. College Station, TX: StataCorp LLC.

Conclusion Our study demonstrated no access related complications both for TRA and TFA. No significant difference was found in number of vessels catheterised and procedure time. More importantly, there were more foci of MRI DWI restriction, representing SEE, in the TRA group compared to TFA. No patient experienced associated neurological symptoms, with normal neurological examination and all were discharged immediately post recovery period. Despite of the proven benefit of the TRA for cerebral angiography, its widespread use may theoretically lead to increased frequency of embolic events during cerebral angiography, which may be of clinical significance, and this warrants further investigation.

Disclosures V. Carraro do Nascimento: None. H. Rice: 2; C; Stryker, Medtronic. L. De Villiers: 2; C; Stryker, Medtronic.

\section{LB-004 INTRAVENOUS ALTEPLASE HAS DIFFERENT EFFECTS ON THE EFFICACY OF ASPIRATION AND STENT RETRIEVER THROMBECTOMY: ANALYSIS OF THE COMPASS TRIAL}

${ }^{1} \mathrm{M}$ Mokin* ${ }^{2} \mathrm{M}$ Waqas, ${ }^{3} \mathrm{~J}$ Fifi, ${ }^{3} \mathrm{R}$ De Leacy, ${ }^{4} \mathrm{D}$ Fiorella, ${ }^{2} \mathrm{E}$ Levy, ${ }^{2} \mathrm{~K}$ Snyder, ${ }^{5} \mathrm{R}$ Hanel, ${ }^{6} \mathrm{~K}$ Woodward, ${ }^{7} \mathrm{I}$ Chaudry, ${ }^{8} \mathrm{~A}$ Rai, ${ }^{9} \mathrm{D}$ Frei, ${ }^{10} \mathrm{~J}$ Delgado Almandoz, ${ }^{11} \mathrm{M}$ Kelly, ${ }^{12} \mathrm{~A}$ Arthur, ${ }^{13} \mathrm{~B}$ Baxter, ${ }^{14} \mathrm{~J}$ English, ${ }^{15} \mathrm{I}$ Linfante, ${ }^{16} \mathrm{~K}$ Fargen, ${ }^{7} \mathrm{~A}$ Turk, ${ }^{3} \mathrm{~J}$ Mocco, ${ }^{2} \mathrm{~A}$ Siddiqui. ' University of South Florida, Tampa, FL; ${ }^{2}$ University at Buffalo, Buffalo, NY; ${ }^{3}$ Icahn School of Medicine at Mount Sinai, New York, NY; ${ }^{4}$ Stony Brook University, Ston Brook, NY; ${ }^{5}$ Baptist Medical Center, Jacksonville, FL; ${ }^{6}$ Fort Sanders Regional Medical Center, Knoxville, TN; ${ }^{7}$ Prisma Health Upstate, Greenville, SC; ${ }^{8}$ West Virginia University, Morgantown, WV; ${ }^{9}$ Swedish Medical Center, Englewood, CO; ${ }^{10}$ Abbott Northwestern Hospital, Minneapolis, MN; ${ }^{11}$ University of Saskatchewan, Saskatoon, SK, Canada; ${ }^{12}$ Semmes-Murphey Clinic, Memphis, $T N_{;}{ }^{13}$ Lehigh Valley Health Network, Allentown, PA; ${ }^{14}$ California Pacific Medical Center, San Francisco, CA; ${ }^{15}$ Baptist Cardiac and Vascular Institute, Miami, FL; ${ }^{16}$ Wake Forest Baptist Medical Center, Winston-Salem, NC

\subsection{6/neurintsurg-2021-SNIS.245}

Background There is conflicting evidence on the utility of intravenous (IV) Alteplase in patients with emergent large vessel occlusion treated with mechanical thrombectomy (MT). The goal of this study was to examine whether there were differences in procedural and technical outcomes of MT depending on whether patients received IV Alteplase prior to intervention using the COMPASS dataset.

Methods This was a post hoc analysis of the COMPASS: A trial of aspiration thrombectomy versus stent retriever thrombectomy as first-line approach for large vessel occlusion. We compared clinical, procedural and angiographic outcomes of patients with and without prior IV Alteplase administration. In order to compare outcomes in patients treated with MT according to the use of IV Altepase, only patients presenting to the emergency department (ED) of the first hospital of arrival within 4 hours of stroke symptom onset or last known normal were included in our analysis.

Results Of the 270 patients included in the COMPASS trial, $235(87 \%)$ arrived to the ED within the first 4 hours of stroke symptom onset and were eligible for the post-hoc analysis. (115 patients in the aspiration first and 120 in the SR first groups, respectively).Atrial fibrillation was more frequently seen in the MT only when compared to the combined group (65.5\% vs $38.6 \%$, respectively, $\mathrm{P}<0.001)$. Other vascular risk factors, age, NIHSS severity, and the distribution of occlusion sites showed no statistically significant difference. On univariate analysis, administration of IV Alteplase prior to MT was found to be significantly associated with favorable outcomes (modified Rankin scale [mRS] $0-2$ at 3 months; $55.6 \%$ vs $40 \%$ in the MT only group, $\mathrm{P}=0.037)$. However, on multivariate analysis only baseline (pre-stroke) mRS, admission NIHSS score and age were identified as independent predictors of favorable outcomes at 3 months. We found higher final TICI $2 b / 3$ rates in patients without the use of Alteplase prior to the aspiration first approach $(100 \%$ vs $87.9 \%$ in IV Altepase + aspiration first $\mathrm{MT}, \mathrm{P}=0.03)$. In the $\mathrm{SR}$ first group, final TICI $2 \mathrm{~b} / 3$ rates were identical in patients with and without IV Alteplase administration $(87.5 \%$ and $87.5 \%$, $\mathrm{P}=1.0)$.

Conclusions Prior administration of IV Alteplase is not associated with improved procedural or clinical outcomes in patients with anterior circulation ELVO treated with MT. Alteplase may adversely affect the efficacy of aspiration first approach, but does not seem to influence the stent retriever first approach to MT.

Disclosures M. Mokin: 1; C; NIH R21NS109575. 2; C; Cerenovus, Medtronic. 4; C; Brain Q, Endostream Serenity medical, Synchron. M. Waqas: None. J. Fifi: 2; C; Stryker, Penumbra, Microvention. 4; C; Cerebrotech, The Stroke Project. R. De Leacy: 2; C; Penumbra, Cerenovus, Siemens, Imperative Care. 4; C; Synchron, Endostream, Q’Apel. D. Fiorella: 2; C; Balt USA, Penumbra, Cerenovus, Stryker, Genentech, Shape Memory Medical, Marbelhead, Medtronic, MENTICE, Neuorogami, RAPID AI, Rapid medical, Phenox. E. Levy: 2; C; Penumbra, NextGen Biologics, Rapid Medical, Cognition Medical, Three Rivers Medical, Stryker, MedX, Endostream Medical. K. Snyder: 2; C; Penumbra, Canon Medical Systems, Medtronic, Jacobs Institute, Neurovascular Diagnostics. R. Hanel: 2; C; Penumbra, Endostream, Cerebrotech, Synchron, InNeuroCo, Medtronic, Microvention, Stryker, Cerenovus; Elum, Three Rivers. K. Woodward: None. I. Chaudry: 2; C; Medtronic, Microvention, Penumbra. 4; C; Cerenovus, Serenity Medical, Cerebrotech, Three Rivers Medical, Q'apel. A. Rai: 2; C; Penumbra, Microvention, Stryker. D. Frei: 2; C; Penumbra, Cerenovus, Stryker, Genentech, Shape Memory Medical, Siemens. J. Delgado Almandoz: 2; C; Penumbra, Medtronic. M. Kelly: 2; C; Penumbra, Medtronic, Endostream. A. Arthur: 2; C; Cerenovus, Medtronic, Microvention, Penumbra, Scientia, Siemens, Stryker. 4; C; Bendit, Cerebrotech, Endostream, Magneto, Marblehead, Neurogami, Serenity, Synchron, Triad, Vascular Simulations. B. Baxter: 2; C; Penumbra , Medtronic, Stryker, Cerenovus, Viz. ai, 880 medical, Route 92, Artio Medical. J. English: 2; C; Penumbra, Medtronic, Stryker, Route 92 Medical. I. Linfante: 2; C; Penumbra, Medtronic, Stryker, Microvention, InNeuroCo, Three Rivers. K. Fargen: None. A. Turk: 2; C; Cardinal consulting, Cerenovus, Corindus, Medtronic, Siemens, 880 medical. 4; C; Cerebrotech, Endostream. Imperative care, Three rivers medical, Vastrax, Shape memory, Synchron, Serenity medical, Blink TBI, Echovate, RIST, Apama, Q’Apel, VizAi, Early bird medical, Rapid medical, S. J. Mocco: 2; C; Imperative Care, Cerebrotech, VIseon, Endostream, Vastrax, RIST, Synchron, Viz.ai, Perflow and CVAid. 4; C; Cerebrotech, Imperative Care, Endostream, Viseon, BlinkTBI, Serenity, Cardinal Consulting, NTI, RIST, Viz.ai, Synchron. A. Siddiqui: 2; C; Amnis Therapeutics, Apellis Pharmaceuticals, Inc., Boston Scientific, Canon Medical Systems USA, Inc., Cardinal Health 200, LLC, Cerebrotech Medical Systems, Inc., 